\title{
The role of residual elastic stress in shrinkage of cold-pressed and sintered fuel pellets
}

\author{
Yuriy Perlovich ${ }^{\mathrm{a}^{*}}$, Margarita Isaenkova ${ }^{\mathrm{b}}$, Olga Krymskaya, \\ Vitaliy Baranov, Mikhail Perlovich, Andrey Tenishev \\ National Research Nuclear University "MEPhl”, Moscow, Russia

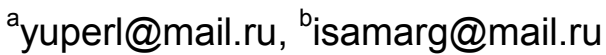

Keywords: fuel tablets, uranium dioxide, sintering, shrinkage, plastic deformation, texture.

\begin{abstract}
Manufacture of fuel pellets from $\mathrm{UO}_{2}$ powder includes many processes, among which shrinkage without application of external loading till now requires explanations. Particles of $\mathrm{UO}_{2}$ are brittle up to $\sim 700^{\circ} \mathrm{C}$. By cold pressing due to the porous structure of powder pellets separate particles are pressing one into another with accompanying rise of residual elastic stress. Under following heating and sintering plasticization of $\mathrm{UO}_{2}$ occurs, its yield stress decreases and conditions for creep and resulting shrinkage of pellets takes place due to initiation of slip by planes $\{001\}$. By heating of pellets their packing texture turns into the sintering texture, which further passes in the texture of dynamic recrystallization. Presented curves show that for complete relaxation of residual stress in cold-pressed pellets the long time is required.
\end{abstract}

\section{Introduction}

Compaction of $\mathrm{UO}_{2}$ powders is an important technological procedure, which in many respects predetermines the work effectiveness of heat-generating elements in nuclear reactors. However, though this procedure is used for many years already, the physical essence of accompanying processes up to now needs to be ascertained. The theory of sintering, developed more than 70 years ago [1], bases predominantly on diffusion models, considering migration of atoms between two spherical grains. But this theory absolutely does not take into account the fact, that finally these grains by some way acquire the same crystallographic orientation. At that, the experimental study of produced pellets did not include X-ray investigation of their structure features. This is a reason for the one-sided interpretation of considered processes.

In the given paper our attention first of all was paid to crystallographic mechanisms of shrinkage as applied to heated cold-pressed pellets, resulting in decrease of the pellet volume by dozens of percents. By obtained X-ray data typical for plastic deformation processes take place in pellets at different stages of their production. In particular, among these processes there is texture formation. Shrinkage of pellets includes transfer of their material by macro-distances at sintering temperatures and at the same time is connected with continuation of texture development. Therefore it is rather evident, that shrinkage is conjugated with plastic deformation of uranium dioxide as well. A question arises concerning a motive power of plastic deformation in pellets under heating without external loading. In order to answer this question, it is necessary to consider successive stages of pellet production and attendant evolution of $\mathrm{UO}_{2}$ properties. The new conception of shrinkage as applied to sintered fuel pellets from uranium dioxide is stated on the basis of experimental data, obtained by methods of X-ray structure and texture analysis.

Manufacture of tablets from uranium dioxide, studied in the given work, corresponded to the standard technology and did not include any fundamental novations [2]. Therefore by consideration of successive stages of this process we pay our attention only to those experimental data, which could not be obtained earlier, since previous investigators, as a rule, did not use methods of X-ray structure and texture analysis. Among them - determination of the X-ray line true angular halfwidth $B_{0.5}$ [3] and construction of inverse texture pole figures by curves of sample tilt [4]. The 
method [3] is used everywhere, at that the angular half-width of X-ray line $B_{0.5}$ is an indicator of substructure condition for reflecting grains, i.e. their distortion $\Delta d / d$, where $d$-interplanar spacing, and coherent block size $D$. The wider is $B_{0.5}$, the higher is strain hardening of material and the level of residual microstress. The method [4], elaborated by authors, differs from the standard one with high precision, statistical significance and expressivity, so that it can be used for revelation even insignificant texture changes. By X-ray study a diffractometer BRUKER D8 DISCOVER was used.

Studied pellets of uranium dioxide were obtained by the procedure, including (a) cold pressing and (b) sintering by a number of regimes. Some pellets after cold pressing were heated at once up to $1600^{\circ} \mathrm{C}$ and kept at this temperature during different time up to $500 \mathrm{~min}$, whereas other pellets were submitted to stepped heating up to temperatures $455^{\circ}-1100^{\circ} \mathrm{C}$ (fig. 1). Sintering of pressed tablets was conducted using a dilatometer NetzchDIL402C by the regular program, modeling regimes of $\mathrm{UO}_{2}$ sintering in real industrial furnaces. The model sintering regime consists of several linear and isothermal regions. According to obtained dilatometric data, sintering of studied pellets at $1600^{\circ} \mathrm{C}$ causes their shrinkage by height $\left(\mathrm{d} L / L_{0}\right)$ up to $20 \%$, whereas sintering by stepped regimes - up to $5 \%$.

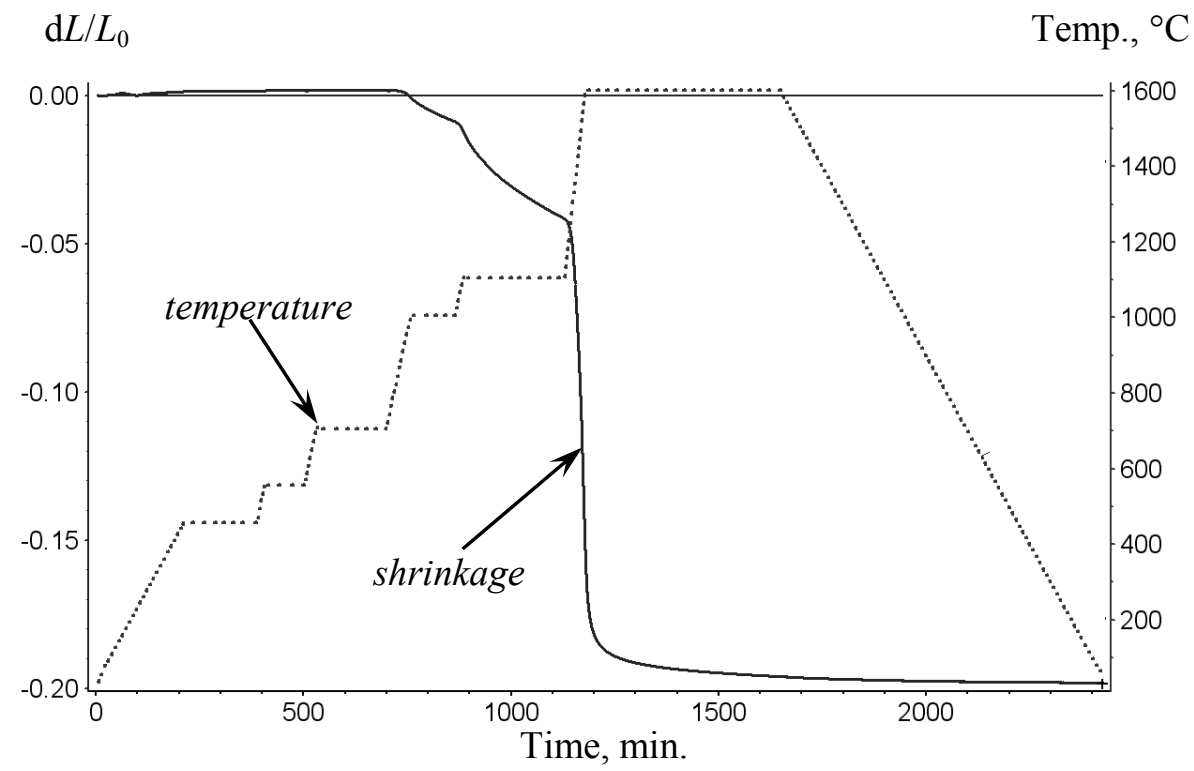

Fig. 1. Dilatometric curve of sintering according to regular program of stepped heating

\section{Cold pressing of pellets}

Independently of the method, used for production of the initial $\mathrm{UO}_{2}$ powder, its particles are in brittle condition right up to temperatures $\sim 600^{\circ} \div 700^{\circ} \mathrm{C}$ and plasticize by their heating above these temperatures. As a result of fragmentation in a mill particles of uranium dioxide destroy by cleavage planes $\{111\}$ [5]. Same cleavage planes are in all fluorites, since namely these planes are separated from neighboring planes with layers of oxygen atoms (fig. 2).

It was stated for the first time that by cold pressing in pellets from uranium dioxide formation of crystallographic texture takes place. It becomes evident from formation of a clear maximum near axis [011] with maximal pole density above 1.2 within elementary stereographic triangle (fig. 3). This signifies, that in the studied sample the number of grains, having such orientation near the pellet axis, is by 1.2 times more, than in the textureless sample. Though this parameter shows, that the texture, formed in the pressed tablets, is not strong, the general character of obtained pole density distribution testifies about quite definite tendency in reorientation of $\mathrm{UO}_{2}$ crystallites by cold pressing of powder. 
The size of $\mathrm{UO}_{2}$ particles after treatment of powder in mill - 200 $\div 300 \mathrm{~nm}$. Pressing of uranium dioxide is carried out usually under pressure of $\sim 200 \mathrm{MPa}$. A single reason, able to condition texture formation in the conglomerate of brittle particles, is mutual slip of these particles by cleavage planes. Then existence of cleavage planes is equivalent to presence of slip planes with a difference that operation mechanisms of these planes by pressing are quite unlike: while mutual slip of brittle particles by cleavage planes has the mechanical character, operation of slip planes has the dislocation nature. Nevertheless, accompanying rotations of crystalline lattice of uranium dioxide particles are similar in both cases and can be described according [6-7]: normal to the slip plane rotates to the axis of compression. At that, as the lattice of crystallite reorients, axis of compression earlier or later proves at the boundary of elementary stereographic triangle and slip by single plane will change into slip by two planes, so that resulting rotations are added together geometrically. In the case of slip by cleavage planes, the axis of pellet, parallel to the axis of compression, coincides with direction [011], as it occurs by compression of metals with FCC crystalline lattice. However, under cold pressing plastic deformation, connected with dislocation slip, is absent in uranium dioxide and the texture, observed in pressed tablets, is a packing texture.

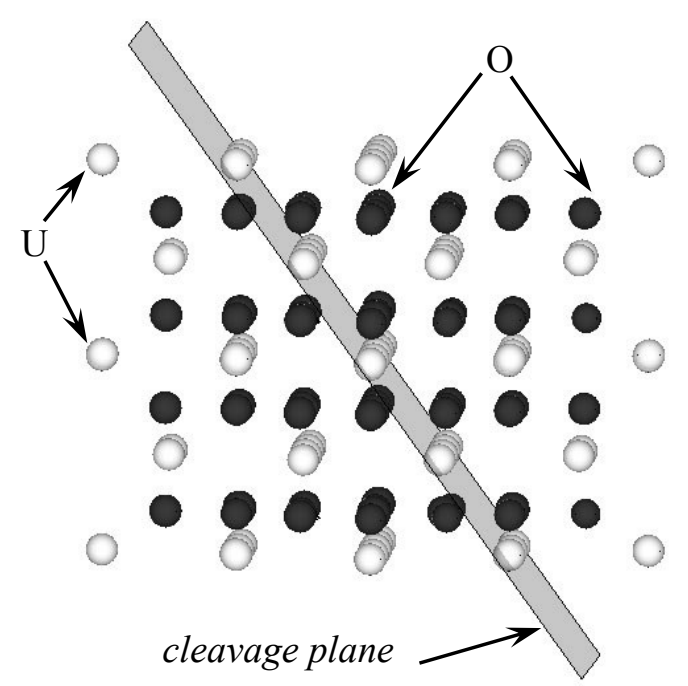

Fig. 2. Position of cleavage planes $\{111\}$, separated from neighboring planes with layers of oxygen atoms in the crystallographic lattice fluorite

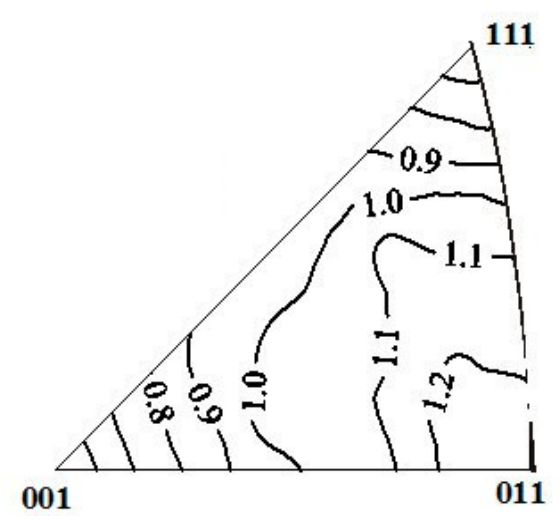

Fig. 3. Inverse texture pole figures of $\mathrm{UO}_{2}$ pellet after cold pressing

\section{Processes in $\mathrm{UO}_{2}$ pellets under sintering}

By heating of pellets, following after their cold pressing, the next series of main processes takes place (some of them are considered in more details in the next section):

1. evaporation of binding substance;

2. thermal expansion of $\mathrm{UO}_{2}$;

3. partial changes of the pellet pressing texture due to rotations of separate powder particles by removal of binding substance and thermal expansion;

4. inhomogeneous brittle - viscous transition in uranium dioxide;

5. local plasticization of $\mathrm{UO}_{2}$ particles and initiation of their creep [8] under influence of residual elastic stress, retaining within some other particles after cold pressing;

6. shrinkage of pellets as a result of pores filling by means of slip under creep deformation;

7. gradual reduction of elastic residual stress;

8. dynamical recrystallization in grains of uranium dioxide, consisting in growth of new grains, differing by their crystallographic orientation from initial ones [9-10];

9. changes of the pellet packing texture by operation of slip systems $\{001\}$ and dynamical recrystallization, conditioned by creep;

10. along with above-noted processes, heating of pressed pellets includes also the complex of diffusion phenomena, which usually are considered as components of properly sintering. 


\section{Residual stress: rise, initiated creep, relaxation}

By cold pressing of brittle powders some diminution of material porosity takes place as compared with that in "piled" condition. In particular, separate particles are pressing in pores between other particles, causing their elastic deformation. Just due to porosity of powder material its pressing is accompanied by rise of significant residual elastic stress. By following heating of pellets the brittle-viscous transition takes place in uranium oxide particles. As a result, these particles become more plastic, their yield point decreases and can prove even lower than the level of residual elastic stress, induced in pellet by cold pressing. But this transition is not momentary - it is extended by temperature, time and space. Whereas some fragments of pellet become already plastic, its another fragments so far are brittle and retain the high level of elastic residual stress. Thus, the inner region of pellet due to the relatively low thermal conductivity of porous ceramic material heats up more slowly, than surface layers. Therefore elastic residual stress, located mainly inside the pellet, can influences deformation of plasticized surface layers. Powder surface particles, which earlier were in brittle condition, now under the influence of residual elastic stress can continue their plastic deformation in the direction of previous cold pressing.

Heating of pellets is realized by absence of external loading, and only internal residual stress influences processes of shrinkage. At that, a value of this residual stress is not higher than the yield point of uranium oxide. Under such conditions it would be justified to consider the observed shrinkage as a result of creep. Plastic deformation, induced by low or even scaling down stress, requires a prolonged time, comparable with duration of heating steps in used sintering regimes. As it is known [8], mechanisms of creep are diverse and, besides diffusion processes, include also dislocation ones. In particular, low-temperature creep operates in uranium dioxide within temperature interval $\sim 800^{\circ}-1200^{\circ} \mathrm{C}$, that is at $\mathrm{T}<\mathrm{T}_{\text {melt }} / 3$, where $\mathrm{T}_{\text {melt }}=3120 \mathrm{~K}$. This signifies, that by indicated temperatures the creep initiates in sintered pellets processes of crystallographic slip, lying in the basis of usual plastic deformation. Just processes of slip are responsible for changes of the cold pressing texture.

Changes of the initial packing texture, formed in pellets by cold pressing, quite clearly testify about participation of plastic deformation in formation of pellet structure at the beginning of sintering. After plasticization of uranium dioxide the slip in its particles develops by systems $\{001\}<011>$, as in other materials with crystalline lattice of fluorite [11]. Sintering of pressed pellets under influence of creep, initiated by residual elastic stress, results in operation of crystallographic slip with accompanying reorientation of $\mathrm{UO}_{2}$ grains to new positions in the stereographic triangle. These new positions can be found using the simplest geometric model of texture formation [6-7]: the texture maximum, corresponding to orientation of pellet axis, becomes more regular, than in pressed pellets, and approaches to the boundary [001-111] (fig. 4).

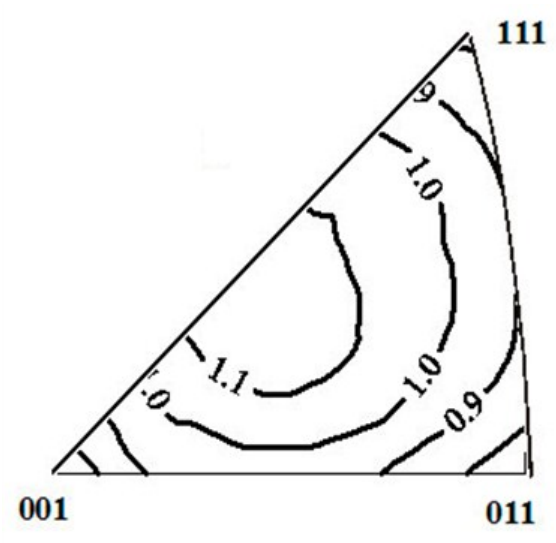

Fig. 4. Typical inverse texture pole figure of sintered $\mathrm{UO}_{2}$ pellet, experienced shrinkage as a result of slip by system $<001>\{011\}$

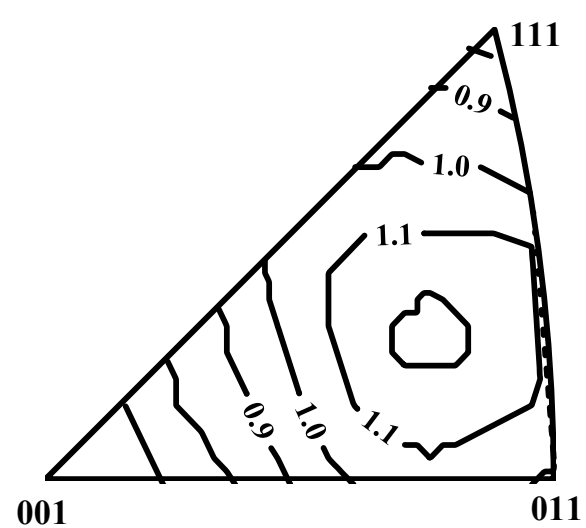

Fig. 5 Typical inverse texture pole figure of $\mathrm{UO}_{2}$ pellet after dynamic recrystallization 
At later sintering stages the superposition of shrinkage and growth of new grains by dynamic recrystallization takes place. Though these processes cannot be clearly differentiated by texture pole figures, but it is doubtless, that deformation by creep is responsible for both. Observed difference of obtained sintering textures indicates, that dynamic recrystallization is much less regular process, than plastic deformation, - therefore texture of sintering often deflects from the predicted one for the case of plastic deformation (fig. 5).

As it is seen in presented pole figures, textures of pellets are relatively weak. However it is understandable, when taking into account, that both shrinkage and plastic deformation cover only a part of pellet, occupied earlier by pores. Therefore sintering without external loading does not affect inner regions of $\mathrm{UO}_{2}$ particles, including their crystallographic orientations. But filling of pores occurs at the expense of particle surface regions, where the brittle-viscous transition takes place first of all.

Among specialists, not implicated in X-ray structure studies, an idea is wide-spread, that residual elastic stress, originated in material by cold treatment, relaxes at once after beginning of its heating. However, this is not so. The fact is that in the conglomerate of mutually misoriented grains their inevitable real structure anisotropy hinders from the stress relaxation. Because of the structure anisotropy thermal expansion coefficients of neighboring grains prove to be mutually disagreed, promoting rise of new microstress. Expected removal of residual stress occurs only by sufficiently high temperatures and duration of heat treatment.

In particular, relaxation of residual stress in cold-pressed $\mathrm{UO}_{2}$ pellets does not take place at once. This is confirmed also by dependences of X-ray lines angular half-width on the temperature of pellet heat time during $150-200 \mathrm{~min}$ at $400^{\circ}-1600^{\circ} \mathrm{C}$ (fig. 6) as well on the duration of their heat time at $1600^{\circ} \mathrm{C}$ (fig. 7), constructed by measurements of this half-width after indicated heat treatments. At that, when judging by slopes of X-ray lines half-width curves in fig. 6 and 7, one can see, that relaxation of residual stress at the closing studied stage of sintering is finished still incompletely.

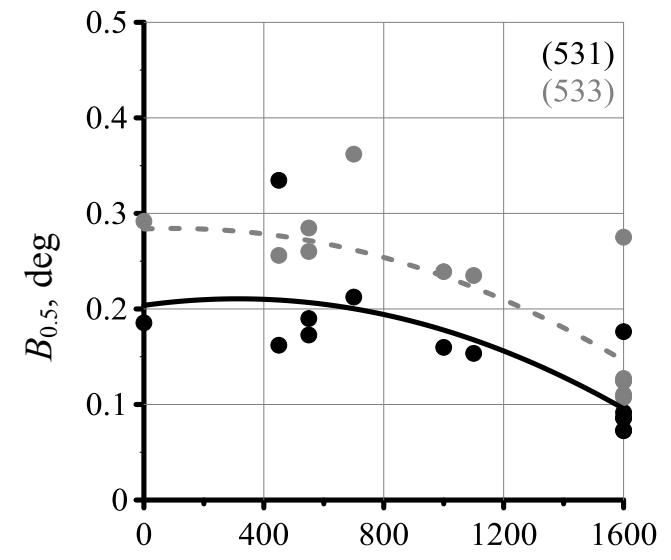

Heating temperature, ${ }^{\circ} \mathrm{C}$

Fig. 6. Dependence ot $\mathrm{X}$-ray I1ne angular halfwidth for sintered $\mathrm{UO}_{2}$ tablets on the temperature of their heat time during 150- $200 \mathrm{~min}$

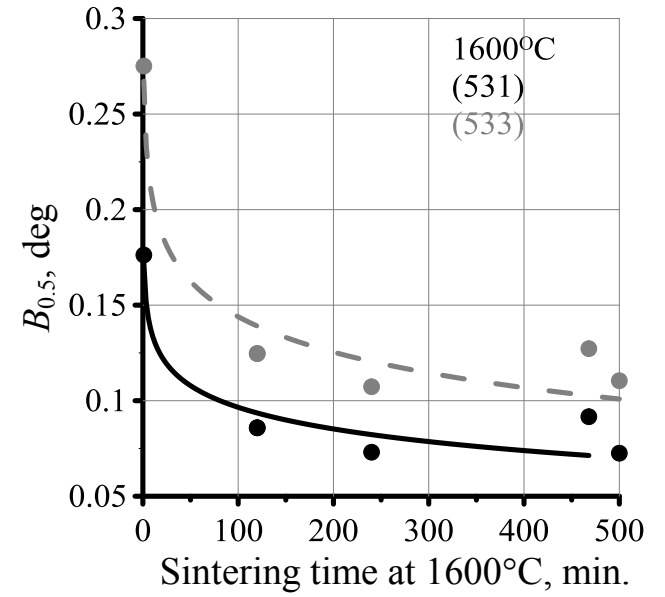

Fig.7. Dependence of X-ray line angular halfwidth for sintered $\mathrm{UO}_{2}$ tablets on their heating time at $1600^{\circ} \mathrm{C}$

\section{Conclusions}

(1) In pellets of uranium dioxide under cold pressing the packing texture forms due to grain shifts by cleavage planes $\{111\}$, and resulting predominant alignment of pellet axes is near the top of stereographic triangle $<011>$.

(2) Shrinkage of pellets by their heating occurs with participation of plastic deformation owing to the slip by planes $\{001\}$, which becomes possible after brittle-viscous transition under influence of residual elastic stress, causing creep of uranium dioxide. 
(3) By stepped heating of pressed pellets up to sufficiently high temperatures deformation by creep is connected with development of dynamical recrystallization, changing the character of observed texture.

\section{References}

[1] Pines B.Ya., Sintering, creep, relaxation, recrystallization and other phenomena caused by selfdiffusion in the crystalline solids. Uspekhi Fizicheskikh Nauk, vol. 52, № 4 (1954) 501-559 (in Russian).

[2] Maiorov A.A., Braverman K.B. Technology of ceramic uranium dioxide production, Energoatomizdat, Moscow, 1985 (in Russian).

[3] A. Taylor, X-Ray Metallography. John Wiley \& Sons, Inc., New York - London, 1961.

[4] Perlovich Yu. A., Isaenkova M. G., Kaplii S. N. Rapid Method of Mapping the Inverse Pole Figures, Industrial Laboratory, vol. 79, № 05 (2013) 27-30.

[5] Abramowski M., Grimes R.W., Owens S. Morphology of $\mathrm{UO}_{2}$, Journal of Nuclear Materials, 275 (1999) 12-18.

[6] Calnan E.A., Clews C.J, Philosophical Magazine B., vol. 41, 7th series, No 322 (1950) 10851100.

[7] Wassermann G., Grewen J., Texturen metallischer Werkstoffe, Springer-Verlag, Berlin Heidelberg, 1962.

[8] Rosenberg V.M., Creep of metals, Metallurgy, Moscow, 1967 (in Russian).

[9] Gorelik S.S., Dobatkin S.V., Kaputkina L.M. Recrystallization of metalls and alloys, 3d ed., MISIS, Moscow, 2005 (in Russian).

[10] Haessner F., Recrystallization of metallic materials, Verlag, Stuttgart, 1978.

[11] Skvorcova N.P., Krivandina E.A., Karimov D.N. Physics of the Solid State, vol. 50, № 4 (2008) 639-643. 